\title{
Complicações ortopédicas na síndrome de Klippel-Trenaunay
}

\author{
Orthopedic complications in Klippel-Trenaunay syndrom
}

Sergio Mainine', Lucas Leite Ribeiro² Thiago Francisco Nascimento², Eiffel Tsuyoshi Dobashi'

\section{Resumo}

Introdução: A Síndrome de Klippel-Trenaunay é uma afecção congênita da mesoderme, infrequente, a qual se caracteriza por uma tríade composta por hemangiomas cutâneos, veias varicosas e hipertrofia dos tecidos ósseos e/ou das partes moles; quando combinada com fístulas arteriovenosas passa a ser denominada Klippel-Trenaunay-Weber-Parkes. Relato de caso: Os autores apresentaram um paciente portador da síndrome de Klippel-Trenaunay associada à deformidade angular de joelho, geno valgo, e gonartrose. Discussão: Em virtude da necessidade do diagnóstico precoce dessa afecção, com a finalidade de prevenção de sequelas,foram apresentados os resultados dos exames imagem para avaliação pré-operatória e a metodologia terapêutica adotada. 0 tratamento consistiu da realização de osteotomia supracondiliana do fêmur, com cunha medial de subtração.

Palavras-chave: Doença Klippel-Trenaunay; genu varum; osteoartrose gonartrose.

\begin{abstract}
Introduction: The Klippel-Trenaunay syndrome is a mesoderm congenital disorder, infrequent, which is characterized by a triad of cutaneous hemangiomas, varicose veins, bone and soft tissues hypertrophy; when associated with arteriovenous fistulas it is called Klippel-Trenaunay-Weber-Parkes. Case report: The authors presented a case report of a patient with Klippel-Trenaunay syndrome associated to angular deform. The authors presente a case report of a patient with Klippel-Trenaunay syndrome associated to angular deformity of the knee, genu valgus and knee arthritis. Discussion: In view of the need for early diagnosis of this condition, in order to prevent sequelae, are presented the results of the image exams to preoperative evaluation and the methodology adopted therapy. The treatment consisted of supracondylar osteotomy. The treatment consisted of the succed of femur supracondylar osteotomy with medial wedge subtraction.
\end{abstract}

Keywords: Disease Klippel-Trenaunay; genu varum; osteoarthritis.

Recebido: 30/8/2009

Revisado: $11 / 12 / 2009$

Aprovado: $16 / 2 / 2010$

\section{Introdução}

A tríade composta por malformações capilares (principalmente os nevus vinhosos), hipertrofia óssea, tecidos moles e veias varicosas ou malformadas ${ }^{1,2}$ caracteriza uma condição patológica congênita rara de etiologia indefinida ${ }^{2}$ denominada Síndrome de Klippel-Trenaunay (SKT) descrita em 19001. Como sinônimos da doença, são comumente utilizados os termos hipertrofia angiectásica congênita, síndrome da osteo-hiperplasia angiectásica, síndrome da osteo-hiperplasia angiectá- sica infantil, síndrome da osteoangiohipertrofia e síndrome de KlippelTrenaunay-Weber (SKTW).

Cabe ressaltar, entretanto, que a associação das fístulas arteriovenosas à SKT, descrita por Parkes Weber em 1907 e 1918, caracteriza-se por manifestações hemodinâmicas variáveis e determina tratamento e prognóstico distintos 3 .

São descritas associações com anomalias do sistema linfático e das veias profundas (atresia, hipoplasia, incompetência vascular e dilatações aneurismáticas $)^{2,4}$, microfístulas arteriovenosas ${ }^{(1,4,5)}$, hemangiomas

Trabalho realizado no Hospital Estadual Mário Covas, Santo André (SP), Brasil.

Grupo de Joelho do Hospital Estadual Mário Covas da Fundação do ABC, Santo André (SP), Brasil

2 Médicos Ortopedistas do Hospital Instituto de Fraturas, Ortopedia e Reabilitação (IFOR), São Bernardo do Campo (SP), Brasil

Endereço para correspondência: Sergio Mainine - Hospital Estadual Mário Covas - Rua Doutor Henrique Calderazzo, 321 - CEP $09190-615$ - Santo

André (SP), Brasil - e-mail: sergiomainine@terra.com.br 
viscerais e faciais, síndrome de Kasabach-Merrit ${ }^{6}$, coagulopatia intravascular disseminada, lipodistrofia, retardo mental ${ }^{7}$, hiperidrose, atrofia da pele, verrugas, dermatites, tromboflebites e celulites ${ }^{8}$.

A SKT acomete invariavelmente os membros superiores e mais raramente o tronco, a cabeça ou o pescoço'; porém, na maioria dos casos, aproximadamente $90 \%$, os membros inferiores são acometidos. O início do reconhecimento da hipertrofia óssea e dos tecidos adjacentes varia consideravelmente, pois pode ser detectada desde o nascimento ou percebida durante o desenvolvimento da criança acometida por esta condição.

Entre as manifestações do sistema musculoesquelético, descritas na literatura ortopédica, são referidos: a displasia do desenvolvimento do quadril (DDQ), a sindactilia, a macrodactilia ${ }^{2,9}$, a polidactilia ${ }^{9}$, o pé metatarso varo, o pé torto congênito o equino-cavo-varo, a anisomelia, a escoliose e as deformidades angulares de membro inferiores.

\section{Relato do caso}

Paciente do sexo masculino, 43 anos, branco, mestre de obras, queixava-se desde a infância de deformidade progressiva em joelho esquerdo. Decidiuprocurar o Ambulatório de Joelho do Hospital IFOR de São Bernardo do Campo, por sentir aumento progressivo de dor no joelho e na região lombar.

Após exame clínico foram percebidos à inspeção: distribuição assimétrica da pilificação, aumentada no hemicorpo esquerdo, mácula rosácea no hemitórax esquerdo, veias varicosas acentuadas em membro inferior esquerdo, hemi-hipertrofia à esquerda, escoliose toracolombar sinistro-côncava redutível e geno valgo acentuado à esquerda.

Os exames radiográficos simples de joelho esquerdo demonstraram um eixo femorotibial com valgo de $20^{\circ}$, valgismo entre a metáfise e diáfise

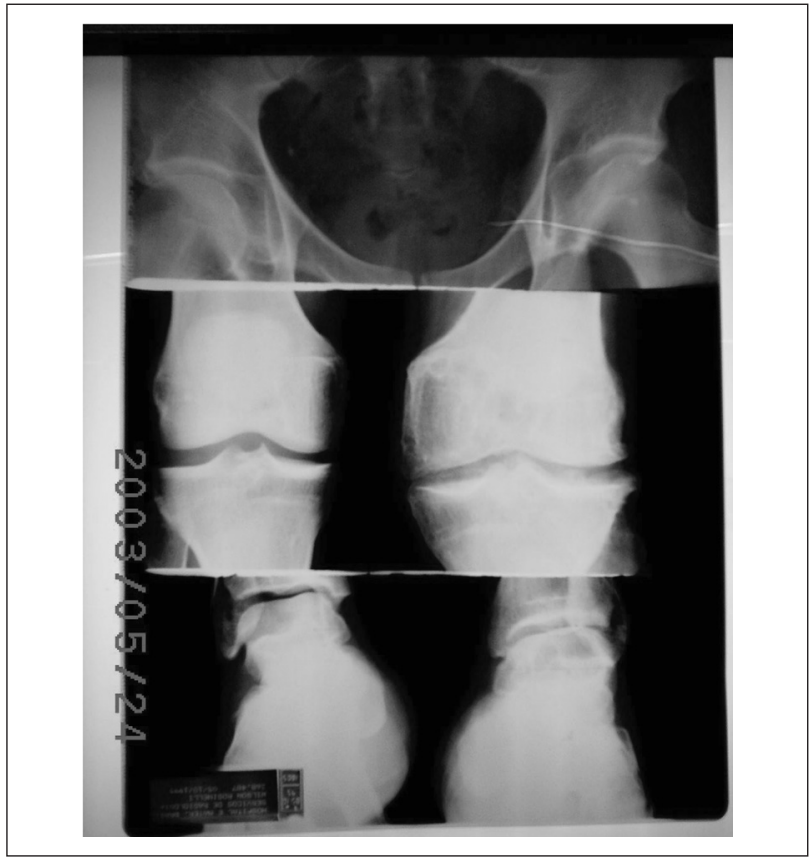

Figura 1 - Escanograma: alongamento do membro inferior esquerdo. da tíbia de $10^{\circ}$, osteofitose intensa patelofemoral, fêmoro-tibial medial e lateral, e uma irregularidade dos côndilos femorais, tibiais e patelofemoral. A escanometria demonstrou um fêmur esquerdo maior que o direito em 2,8 cm, e tíbia esquerda maior do que a direita em 1,7 cm, num total de 4,5 cm de discrepância entre os membros inferiores (Figura 1).

Não houve alteração arterial demonstrável à arteriografia; no entanto, foi observada ausência de contrastação da veia femoral esquerda e de vávulas do lado esquerdo, com varizes exuberantes, durante flebrografia (Figura 2).

Consequentemente, após investigação foi realizado o diagnóstico de SK T associado a geno valgo e escoliose.

Foi iniciado um processo de investigação e avaliação pré-operatória, baseado principalmente na preocupação da ocorrência de maior sangramento intraoperatório e até hemorragias. As perspectivas de alterações cicatriciais reconhecidas na SKT também foram consideradas.

Após essa investigação e rigorosa avaliação pré-anestésica, o paciente foi considerado pronto para submeter-se ao tratamento operatório, que consistiu em osteotomia supracondiliana do fêmur esquerdo, com cunha medial de subtração clássica (Figura 3).

Em reavaliação pós-operatória de três anos, o paciente apresentou-se com membros inferiores (MMII) alinhados, sem queixas de dor no joelho e na região lombar (Figura 4).

\section{Discussão}

Em 1900, os médicos franceses Klippel e Trenaunay descreveram uma síndrome caracterizada por: hemangiomas de extremidades (malformações capilares), hipertrofia de ossos e tecidos e presença de varizes ou malformações venosas ${ }^{1}$. Klippel e Trenaunay ${ }^{1}$ não mencionaram

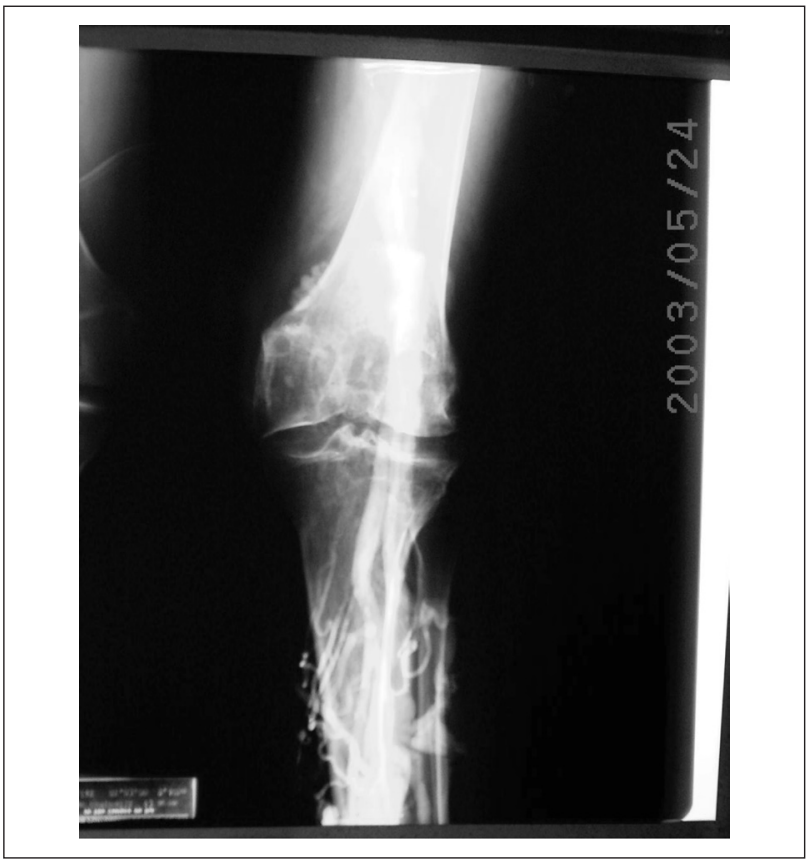

Figura 2 - Flebografia: varizes em membro inferior esquerdo. 
fístulas arteriovenosas. Ao contrário, Parkes-Weber ${ }^{3}$ chamou a mesma síndrome de hipertrofia hemangiectásica com dilatações arteriovenosas (fístulas). Ainda existe confusão acerca dessas duas síndromes. Entretanto, esses autores acreditam que as diferenças entre elas são caracterizadas pela existência ou não de fístulas arteriovenosas, o que implica diferenças terapêuticas e prognósticas.

Na SKT, os membros inferiores são os mais acometidos (85\%) e o acometimento unilateral o mais frequente ( $85 \%)$. Membros superiores (15\%), ambos os membros (10\%) ou forma cruzada são acometimentos mais raros. O hemangioma pode estar presente do nascimento aos seis anos sendo que a maioria dos sintomas está presente antes do primeiro ano ${ }^{8}$. Na maioria dos casos a manifestação clínica da doença é notada ao nascer ou nos primeiros anos de idade ${ }^{6}$.

Hemi-hipertrofia e hemi-hipotrofia são definidas como assimetria entre os lados esquerdo e direito do corpo, numa proporção muito maior do que poderia ser considerado normal. Pode envolver o comprimento ou a circunferência dos membros, assim como a cabeça, o tronco e os órgãos internos. Deve-se, ao examinar, procurar malformações vasculares associadas a malformações dos dedos ou macrodactilia, as quais normalmente estão associadas a condições de aumento do crescimento. Musculatura hipotrofiada, alterações neurológicas, retardo mental ou anormalidades de movimentos podem estar associadas ao hipocrescimento.

A ressonância magnética e outras técnicas de imagem podem determinar a variação normal de discrepância nos membros, definida como assimetria anormal ( $5 \%$ de diferença entre os membros), seja em comprimento ou em circunferência.

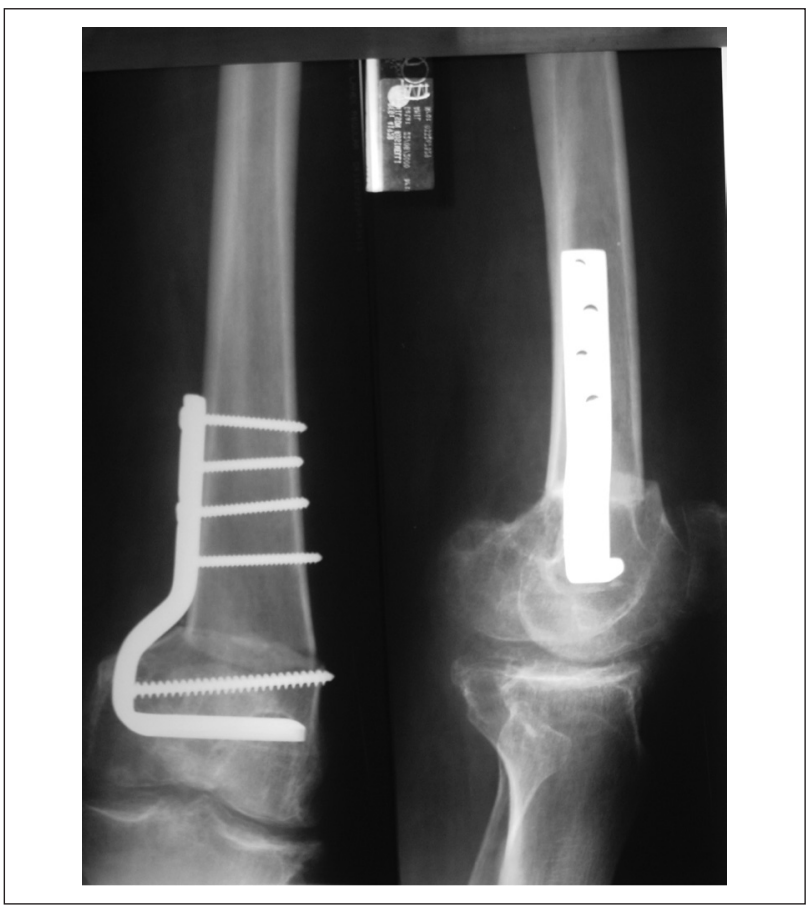

Figura 3 - Pós-operatório: correção do genovalgo.
A hemi-hipertrofia e a hemi-hipotrofia podem ser inicialmente classificadas em congênitas ou adquiridas. A assimetria adquirida pode resultar de trauma, infecção, radiação ou inflamação. As formas congênitas podem ser classificadas como total ou limitadas, sindrômicas (parte de uma síndrome) ou não-sindrômicas (isoladas).

O hipercrescimento pode ser idiopático ou associado à hipertrofia não-sindrômica, como na síndrome de Beckwith-Wiedemann, neurofibromatose e Bannayan-Zohana; ou associado a malformações vasculares como SKT, síndrome de Proteus ou malformações linfáticas. A hemi-hipertrofia idiopática ou não-sindrômica está presente em aproximadamente 1 para 50.000 na população. É raro manifestar-se ao nascimento e é mais comum o aparecimento com o crescimento.

Lesões cutâneas vasculares não estão associadas à hemi-hipertrofia não-sindrômica. São raras as anomalias esqueléticas como macrodactilias, displasia do desenvovimento do quadril e pé torto congênito. Anomalias genitourinárias são frequentemente associadas assim como hérnias inguinais, criptorquidia e alterações na medular do rim. Estudos recentes sugerem uma incidência de 5,9\%. Ultrassonografia abdominal é recomendada como screening para crianças com hemi-hipertrofia.

A exata etiologia não está esclarecida'. De acordo com a definição dada por Szilagyi et al. ${ }^{6}$, a síndrome é um misto de malformações vasculares sem fístulas arteriovenosas de grande débito. Dentre as causas sugeridas, o desenvolvimento anormal da mesoderme leva a um aumento no tamanho e número de veias ${ }^{6}$. A hipótese de uma anormalidade da mesoderme pode ser controlada por fatores de angiogênese e vasculogênese como o fator de crescimento endotelial vascular (VEGF) (ou FCEV) ${ }^{6}$. Uma interrupção do delicado balanço do VEGF mediada

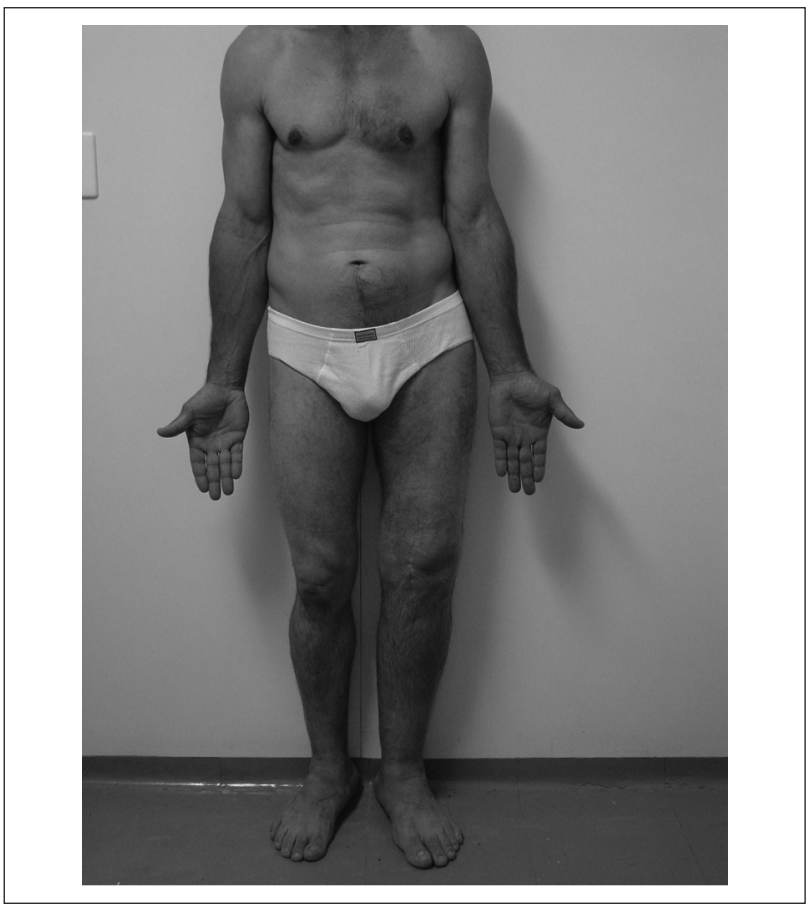

Figura 4 - Hipertrofia e hipertricose do hemicorpo esquerdo: correção do genovalgo 
por uma remodelação vascular pode resultar em um desenvolvimento anormal vascular e consequente hipertrofia do membro ${ }^{6}$.

No entanto, os efeitos dos fatores hemodinâmicos na hipertrofia dos membros ainda não estão confirmados e podem ser menos significantes do que previamente suposto ${ }^{6}$. Alguns autores propõem que o fluxo sanguíneo é maior nos membros afetados, porém a ausência de fístulas arteriovenosas dificulta o esclarecimento da hipertrofia. Os nevus, segunda característica da STK, são decorrentes do aumento da circulação capilar durante o desenvolvimento uterino, sendo essa uma anormalidade da mesoderme ${ }^{2}$.

Alguns autores, após estudarem o efeito da hiperpressão venosa sobre o hipercrescimento, citaram a estase venosa como causa do hipercrescimento do membro e a ligadura da veia do membro contralateral como compensadora desta diferença. Porém, há autores que acreditam que a hipertrofia nada tem a ver com a estase venosa existente no membro hipertrofiado?

A maioria dos casos é esporádica e não foi confirmada herança familiar, embora já tenham sido descritos casos familiares. Em uma série de 47 pacientes portadores da síndrome, foi visto que as mães de dois pacientes tinham hemangiomas capilares e a irmã de outro paciente também tinha hemangioma que foi retirado por razões estéticas ${ }^{10}$. Em uma investigação com 114 pacientes portadores da SKT os autores acreditaram tratar de uma herança multifatorial ${ }^{9}$, porém há quem afirma que o número pequeno da amostra não permite validar tal hipótese?.

A ressonância magnética é o exame de imagem de escolha ${ }^{3}$ para essa complexa síndrome congênita. As desvantagens incluem a menor resolução de imagem se comparada à angiografia com contraste e menos informações a respeito do fluxo sanguíneo. Recomenda-se a realização da ressonância magnética para visibilização de anormalidades venosas e locais de comunicação de sistemas venosos profundos ${ }^{3}$.

Numerosos artigos têm discutido o tratamento cirúrgico desses pacientes ${ }^{1,2,4,5,8,10}$. O consenso mostra que a ligadura ou excisão das varizes não traz benefício e pode piorar os sintomas ${ }^{2,6,8}$.
A maioria dos pacientes é tratada de maneira expectante devido à alta frequência de recidiva $(50 \%)^{6}$. A intervenção cirúrgica nesses pacientes deveria ser restrita à desobstrução de veias profundas e correção de desigualdade de membros inferiores, afirmou Lindenauer em $1971^{7}$. Artigos mais recentes sugerem que a cirurgia ortopédica pode ser mais necessária em outras situações do que na discrepância dos membros inferiores ${ }^{3,6,7,10}$ incluindo cirurgia para diminuição do pé para utilização de calçados e amputações para promover funcionalidade ou melhor aparência ${ }^{7}$.

Em uma série de 47 pacientes, uma menina de oito anos foi submetida a encurtamento e osteotomia derrotativa do fêmur devido ao encurtamento de $4,5 \mathrm{~cm}^{10}$. Antes da intervenção cirúrgica, a flebografia com contraste e a angiorressonância magnética puderam documentar mais detalhadamente a anatomia vascular. $\mathrm{O}$ tratamento cirúrgico venoso mais realizado é a ressecção das veias, incluindo as embriogênicas e excisão das varicosidades. Embora a excisão das varizes seja frequentemente incompleta e as malformações venosas possam recidivar em $50 \%$ dos pacientes, grande melhora clínica é notada na maioria deles? Se agenesia das veias profundas estiver presente, não devem ser indicadas varicectomias extensas ${ }^{8}$.

Quanto à discrepância dos membros inferiores, a epifisiodese da tíbia só é indicada quando a discrepância entre os membros for grande o suficiente para não permitir o uso de palmilhas corretivas ou sapatos adaptados para diminuir a diferença ${ }^{8}$. O tratamento cirúrgico deve ser guiado pelos sintomas 9 . Para os pacientes com sangramento e sintomas persistentes relacionados à hipertensão venosa, a cirurgia deve ser considerada?

Como a SKT é rara e os problemas dos pacientes são complexos, estes devem receber cuidados multidisciplinares em centros qualificados 9 , devendo ter seu diagnóstico sindrômico o mais precoce possível para que se tenha um tratamento mais eficaz.

\section{Referências}

1. Klippel M, Trenaunay P. Du noevus variqueux osteo-hypertrophiques. Archives of General Medicine (Paris). 1900;185:641-72.

2. Baskerville PA, Ackoyd JS, Browse NL. The etiology of the Klippel-Trenaunay syndrome. Ann Surg. 1985;202(5):624-7.

3. Parkes-Weber F. Hemangiectasic hypertrophy of limbs: congenital phlebarteriectasis and so-called congenital "varicoses veins". Br J Child Dis. 1918:15:13-7.

4. Baskerville PA, Ackroyd JS, Lea Thomas M, Browse NL. The KlippelTrenaunay syndrome: clinical, radiological and haemodynamic features and management. Br J Surg. 1985;72(3):232-6.

5. Szilagyi DE, Smith RF, Elliot JP, Hageman JH. Congenital arteriovenous anomalies of the limbs. Arch Surg. 1976;111(4):423-9.
6. Kasabach HH, Merritt KK. Capillary hemangioma with extensive purpura: report of case. Am J Dis Child. 1940;59:1063-70.

7. Stephan MJ, Hall BD, Smith DW, Cohen MM Jr. Macrocephaly in association with unusual cutaneous angiomatosis. J Pediatr. 1975;87(3):353-9

8. Gloviczki P, Hollier LH, Telander RL, Kaufman B, Bianco AJ, Stickler G. Surgical implications of Klippel Trenaunay syndrome. Ann Surg. 1983; 97(3):353-62

9. Noel AA, Gloviczki P, Cherry KJ Jr, Rooke TW, Stanson AW, Driscoll DJ Surgical treatment of venous malformations in Klippel-Trenaunay syndrome. J Vasc Surg. 2000;32(5):840-7.

10. Samuel M, Spitz L. Klippel-Trenaunay syndrome: Clinical features complications and management in children. Br J Surg.1995;82(6):757-61. 